\title{
Édouard Glissant, Poetica della Relazione. Poetica III
}

\section{Alessandro Corio}

\section{(2) OpenEdition}

\section{Journals}

\section{Edizione digitale}

URL: https://journals.openedition.org/studifrancesi/9679

DOI: 10.4000/studifrancesi.9679

ISSN: 2427-5856

\section{Editore}

Rosenberg \& Sellier

\section{Edizione cartacea}

Data di pubblicazione: 1 décembre 2007

Paginazione: 698-699

ISSN: 0039-2944

\section{Notizia bibliografica digitale}

Alessandro Corio, «Édouard Glissant, Poetica della Relazione. Poetica IIl», Studi Francesi [Online], 153 (LI ] III) | 2007, online dal 30 novembre 2015, consultato il 21 septembre 2021. URL: http:// journals.openedition.org/studifrancesi/9679 ; DOI: https://doi.org/10.4000/studifrancesi.9679

Questo documento è stato generato automaticamente il 21 septembre 2021.

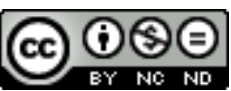

Studi Francesi è distribuita con Licenza Creative Commons Attribuzione - Non commerciale - Non opere derivate 4.0 Internazionale. 


\title{
Édouard Glissant, Poetica della Relazione. Poetica III
}

\author{
Alessandro Corio
}

\section{NOTIZIA}

ÉDOUARD GLISSANT, Poetica della Relazione. Poetica III, traduzione di Enrica RESTORI, Macerata, Quodlibet, 2007, pp. 216.

1 La produzione poetica, teorica e narrativa di Glissant è ancora poco tradotta e diffusa in Italia, per motivi almeno in parte legati alla difficoltà e all'“opacità" dei suoi testi, la cui densità linguistica e concettuale non si arrende neppure al più audace dei traduttori e spaventa un mercato editoriale abituato a prodotti più facilmente commercializzabili.

2 Anche per questi motivi merita di essere sottolineato il coraggio della traduttrice, Enrica Restori, e della casa editrice Quodlibet - che si è già più volte contraddistinta per aver inserito nel suo catalogo dei testi di alto valore letterario e teorico, ma ancora poco conosciuti dal grande pubblico - nel proporre ai lettori italiani quest'opera stilisticamente ibrida, poetica e filosofica al tempo stesso, in cui Glissant rompe volutamente qualsiasi schema formale riconducibile a un ordine espressivo e cognitivo occidentale. Proprio a fronte di una presunta "intraducibilità", la Poetica della Relazione assume valore in quell'ottica di relazione asimmetrica, di tradimento e di supplemento che caratterizza, secondo l'autore, la relazione, la traduzione e la "creolizzazione" di linguaggi e culture nella "totalité-monde".

3 La scrittura di Glissant è, sin dagli esordi (Les Indes, 1956; La Lézarde, 1958), profondamente connessa all'abisso fondatore della "non-storia" caraibica, l'enorme alienazione storica e culturale prodotta dalla colonizzazione europea e, soprattutto, da oltre tre secoli di brutale tratta degli schiavi - "l'olocausto degli olocausti" - nonché dall'apparato di asservimento e di sfruttamento totale dell'essere umano realizzato nel sistema delle piantagioni, esteso dalla Louisiana a Bahia, attraverso quella sorta di "prefazione al continente americano" che è l'arcipelago caraibico. Dall'abisso, dalla 
"permanente condizione ontologica di dolore" (Paul Gilroy, The Black Atlantic, Meltemi, 2003), tradotta metaforicamente da Glissant nella visione della "stiva" della nave negriera - "il ventre stesso della bestia" - risorge quel grido di ribellione e di rifiuto, quella sottrazione che si tradurrà nell'esperienza storica del "marronaggio", ossia nella fuga dello schiavo dall'universo delle piantagioni per rifugiarsi sulle alture nel cuore della foresta e dar vita a comunità di ribelli. Da quel grido mai estinto e dalla traccia sotterranea da esso lasciata nell'inconscio dei popoli della Tratta, custodita dal silenzio delle foreste e dalla voce notturna e ambigua del conteur créole, nasce una parola nuova e soprattutto carica di un sentimento profetico verso le umanità del mondo, quello che Glissant chiama il "pensiero del Tout-monde".

Da questo nucleo storico, linguistico e culturale si generano alcuni dei temi e dei concetti fondamentali della Poetica della Relazione, che appaiono tra di loro intrecciati proprio come quelle radici "rizomatiche" che Glissant, traducendo il concetto metodologico di rizoma elaborato da Deleuze e Guattari in Milles Plateaux, assume come modello identitario contrapposto a quello a "radice unica" che caratterizza invece $\mathrm{i}$ modelli di pensiero "continentali". I "pensieri di sistema", come ha ribadito nella lectio magistralis tenuta al Festival della Filosofia di Roma il 13 maggio 2007, si sono dimostrati, infatti, tanto sontuosi e fecondi per l'Occidente, quanto mortali per quei popoli cui sono stati imposti. L'eco profonda di quel grido originario, al contrario, prende forma traducendosi in un "pensiero dell'erranza e della Traccia", capace di guidarci attraverso una lettura della storia profondamente distante dall'universalismo del progetto dell'umanesimo occidentale e che si pone, a livello dell'elaborazione teorica, agli antipodi del "pensiero dell'Uno" e della violenza epistemica del discorso coloniale.

5 Il discorso teorico, articolato in un movimento spiraliforme in cinque sezioni («Avvicinamenti», «Elementi», «Strade», «Teorie» e «Poetica»), si configura dunque come un'"evasione" dall'Essere inteso come "substans fondativa", mostrando in filigrana una serie di parentele con quella critica della metafisica occidentale elaborata dal post-strutturalismo europeo, con Lévinas, Derrida, Foucault e Deleuze, ma anche con alcuni scrittori e critici postcoloniali come Edward Said, Homi Bhabha e Salman Rushdie. L'originalità della genesi e della formulazione dei concetti in Glissant mantiene comunque una costante e consapevole autonomia rispetto al pensiero eurooccidentale e sorge da un profondo senso della specificità della lotta anticoloniale nei Caraibi. Il pensiero occidentale, secondo Glissant, ha sempre tradotto il proprio impulso universalizzante in un'esigenza di trasparenza, per cui ogni forma di alterità è interpretabile e comprensibile attraverso le istanze della ragione. Questa "volontà di potenza" del logos e il relativo impulso alla "comprensione" dell'altro - dal latino cumpraendere, che ricorda il gesto avvolgente delle braccia e che traduce quindi una forma possessiva di appropriazione dell'alterità - rivelano il nesso profondo tra "volontà di potere" e "volontà di sapere". "Per poterti "comprendere"», afferma Glissant, «e dunque accettarti, devo ricondurre il tuo spessore a quella scala di valori ideale che mi fornisce motivo di paragoni e forse giudizi. Devo ridurre» (p. 173).

6 Da questa prospettiva il poeta martinicano - con un notevole balzo in avanti oltre le politiche del riconoscimento e del rispetto della diversità, facilmente manipolabili dai falsi apologeti del multiculturalismo relativista e spesso prossime a nuove forme di "razzismo postmoderno" - rivendica strenuamente il "diritto all'opacità", ossia a una "divergenza esultante delle umanità" e a una "singolarità non riducibile" che non si 
racchiuda in una sorta di autismo identitario, ma che fondi le basi di un'autentica relazione e di un divenire di scambio continuo con l'Altro: «La trasparenza non appare più come il fondo dello specchio in cui l'umanità occidentale rifletteva il mondo a sua immagine; in fondo allo specchio c'è ora dell'opacità, tutto un limo depositato dai popoli, limo fertile ma, a dire il vero, incerto, inesplorato, ancor oggi [...] negato o offuscato, di cui non possiamo non vivere la presenza insistente». Ed è proprio la presenza "insistente" di ciò che Lévinas ha definito come "la trascendenza del volto dell'altro" che rende possibile quel fenomeno così complesso e carico di imprevedibilità e di ricchezza che Glissant chiama creolizzazione.

7 La "creolizzazione" delle culture nella "totalità-mondo" non si basa dunque sull'appartenenza a un territorio, sull'essenza e sull'esclusione dell'altro e quindi sull'intolleranza, bensì sulla conflittualità e l'imprevedibilità di risultanti della Relazione, sull'erranza e sul multilinguismo. Scrivere oggi, afferma Glissant, significa irrevocabilmente scrivere "alla presenza di tutte le lingue del mondo", anche quando non si conosce e non si parla che la propria. Questa poetica di apertura, questa totalità non totalizzante e priva di assoluto non nasconde gli elementi più problematici di quel processo storico di lunga durata che nominiamo "globalizzazione". La riflessione e lo scandaglio lirico dell'abisso del "bateau négrier", da cui questa poetica sorge, si rovescia nel capitolo iniziale intitolato «La barca aperta», in un'immagine di radiosa bellezza, in un'eco baudelairiana che si fa metafora viva dell'intera poetica di Glissant e risposta al dolore insondabile del proprio passato: “"Salve, antico Oceano!". Preservi sulle tue creste la sorda imbarcazione delle nostre nascite, i tuoi abissi sono il nostro stesso inconscio, solcati da fuggitive memorie. Poi disegni queste nuove rive, noi vi ancoriamo le nostre piaghe striate di catrame, le bocche arrossate e i clamori taciuti. [...] Ci conosciamo, folla, nell'ignoto che non atterrisce. Gridiamo il grido di poesia. Le nostre barche sono aperte, le navighiamo per tutti» (pp. 21-22). 